\title{
DIE NEUE VERFASSUNG VON AFGHANISTAN (1964) UNTER BESONDERER BERÜCKSICHTIGUNG DES RECHTSWESENS
}

\author{
Von Mohammed Ebrahim Modjaz
}

Im Missan 1343 (Oktober 1964) ist eine neue Verfassung für das Königreich Afghanistan in Kraft getreten und hat damit die Verfassung von 1310 (1931) abgelöst. Dieses Ereignis ist insofern höchst interessant und für die Entwicklung des Orients von großer Bedeutung, als es, nach den vorausgehenden Demokratisierungsversuchen, für dieses bis dahin sehr konservativ islamische Land einen gewaltigen Schritt nach vorwärts darstellt, mit dem es viele seiner Nachbarn im Demokratisierungsprozeß überflügelt.

\section{Auswirkungen der neuen Verfassung auf die Entwicklung des Landes unter dem Aspekt der Modernisierung}

Von alters her sind die Menschen fortwährend bemüht, ihren Lebensstandard durch Erfindungen, Entdeckungen und Erneuerungen in Wissenschaft und Technik zu verbessern. Nach dem die Grundsteine der heutigen Wissenschaft und Technik während Tausender von Jahren im Vorderen Orient, in Mittelasien und im Fernen Osten gelegt worden waren ${ }^{1}$, erweiterten die Völker des Westens die Erkenntnisse im Laufe der Jahrhunderte und besonders während der Zeitalter der Renaissance und der industriellen Revolution und führten sie zu einem solchen Höhepunkt, $\mathrm{daß}$ seitens der östlichen und übrigen an dieser Entwicklung behinderten Völker plötzlich das Bedürfnis entstand, zur Verbesserung ihres Lebensstandardes, der nunmehr im Vergleich zum Westen zu niedrig war, wiederum die vervollkommneten Kenntnisse aus dem Westen zurückzuübernehmen.

Am Anfang vollzog sich der Prozeß der „Zurückübernahme“ nur in der gesellschaftlichen Oberschicht und ohne jegliche geistige und körperliche Anstrengung in Form der bloßen Nachahmung und des Verbrauchs der fertig produzierten Güter. Man nannte diesen Prozeß „Europäisierung“ oder „Verwestlichung“. Sehr jung ist jedoch die Einsicht, daß die bloße Nachahmung nur zur augenblicklichen Verschönerung des Lebens führt, daß diese Errungenschaften nicht von langer Dauer sein können und daß es besser wäre, einen tieferen und gründlicheren Einblick in die Herstellung beziehungsweise Entstehung dieser eingeführten materiellen und geistigen Güter zu erwerben. So entstand die Bewegung der „Modernisierung“, die viel weitere Kreise der Bevölkerung erfaßt.

Unter „Modernisierung“ versteht Daniel Lerner „Die Übernahme eines rationalistischen und positivistischen Geistes“. David Apter definiert in einer Studie über die "Politik der Modernisierung“ den Begriff als einen Prozeß der zunehmenden Komplexität in den menschlichen Beziehungen, innerhalb deren die Politik die Hauptrolle spielen soll ${ }^{3}$.

1 Vgl. Will Durant, Die Kulturgeschichte der Menschheit, Lausanne um 1965, Band I, S. 182, 183; Band II, S. $144-146$.

2 Lerner, Modernisierung des Lebensstils, Eine Theorie, in der Sammlung „Soziologie der Entwicklungsländer“, herausgegeben von Peter Heintz, Köln-Berlin 1962, S. 211-243, insbesondere S. $212,213$.

3 Apter, The Politics of modernization, Chikago 1965, S. 3: "The dynamic aspect of modernization for the study of politics can be expressed in the general proposition that modernization is a process of increasing complexity in human affairs within which the policy must act." 
Nach diesen einführenden Worten über die Modernisierung soll untersucht werden, welche Einflüsse die neue Verfassung auf das Leben der Bevölkerung in Afghanistan bereits gehabt hat und welche in der Zukunft zu erwarten sind. In einer Aufzählung der Merkmale der hochindustrialisierten Gesellschaft sieht Peter Heintz ${ }^{4}$ die Modernisierung in einem hohen Grad der Bürokratisierung der Produktionsorganisationen, relativ offenem sozialen Schichtungssystem mit entsprechend relativ breiter Mittelschicht. Daniel Lerner ${ }^{5}$ hält dagegen für die Modernisierung einer Gesellschaft mit niedrigem Lebensniveau, was auch für Afghanistan zutrifft, die Erfüllung folgender Voraussetzungen für notwendig:

1. Urbanisierung,

2. Zunahme des Alphabetentums,

3. Interesse am politischen Geschehen, etwa durch Wahlen,

4. Zugang zu den Massenkommunikationsmitteln Presse, Rundfunk und Film.

In bezug auf die erste Voraussetzung Lerners, Urbanisierung, enthält Art. 26 der neuen Verfassung folgende Bestimmung:

"Jeder Afghane hat das Recht, in jedem Teil seines Staatsgebietes zu reisen und sich dort niederzulassen."

Von diesem Recht der Freizügigkeit, das in der alten Verfassung von 1931 nicht verankert war, wird bereits rege Gebrauch gemacht. Die Errichtung der vielen zunächst kleineren bis mittleren Industrieanlagen lockt die ländliche Bevölkerung in die großen Städte wie Kabul, Kandahar, Herat und Mazar-e-Scharif. Diese Zuwanderung stellt den Staat selbstverständlich vor eine Reihe von Problemen arbeits- und sozialrechtlicher Natur, denen meines Erachtens nicht etwa durch Einschränkung der Zuwanderung, wie Helmut $\mathrm{Hahn}^{6}$ es empfiehlt, sondern durch vermehrte Schaffung von Arbeitsplätzen und Wohnungen begegnet werden sollte.

Die Urbanisierung fördert die zweite Voraussetzung der Modernisierung im Sinne Lerners, nämlich die Zunahme des Alphabetentums ${ }^{7}$. Dieser zweiten Voraussetzung trägt die neue Verfassung in Art. 34 Rechnung:

„Bildung ist das Recht aller Staatsbürger; sie wird unentgeltlich vom Staat beziehungsweise einzelnen Staatsbürgern ${ }^{8}$ ermöglicht. Ziel des Staates ist die Erreichung einer Stufe, in der angemessene Möglichkeiten zur Bildung und Erziehung für alle Bürger bereitstehen. Die Teilnahme am Elementarunterricht ist für alle Kinder in denjenigen Gebieten, in denen die Mittel schon bereitgestellt worden sind, Pflicht."

Die Aufgaben der Bildungsplanung werden hauptsächlich vom Kultusministerium wahrgenommen. Gründung der Elementar-, Volks- und Hochschulen in allen Teilen des Landes sowie die Ausbildung des Lehrpersonals zählt zum Aufgabenbereich dieses Ministeriums.

Die dritte Voraussetzung Lerners, die Förderung des Interesses am politischen Geschehen durch Wahlen, wird in der neuen Verfassung im Kapitel über das Parlament berücksichtigt (Art. 41 bis 77). Art. 41 besagt, daß Afghanistans Parlament die Manifestation des Willens der Bürger ist und das ganze Volk repräsentiert. „Afghanistans Bürger“, so heißt es weiter in Art. 41, „nehmen durch das Parlament am politischen Leben des Landes teil.“ Nach Art. 49 werden Wahlen

4 "Soziologie der Entwicklungsländer“, S. 16.

5 A. a. O., S. $215 \mathrm{ff}$

6 Die Stadt Kabul (Afghanistan) und ihr Umland I, Bonner geographische Abhandlungen 1964, Heft 34, S. 81

7 Vgl. Lerner, a. a. O., S. 227.

8 Bezieht sich auf nicht-allgemeinbildende Schulen, wie z. B. Berufs- und Berufsfachschulen. 
gemäß den Gesetzesvorschriften abgehalten. Auf Grund dieser Bestimmung der Verfassung ist das Parlamentswahlgesetz von 1965 (55 Paragraphen) erlassen worden, das auch der Frau, die noch bis 1959 der isolierenden Wirkung des Schleiertragens in der Offentlichkeit ausgesetzt war, das aktive und passive Wahlrecht zubilligt.

Die vierte Voraussetzung Lerners, der Zugang zu den Massenkommunikationsmitteln, wird von der neuen Verfassung in Kapitel 3 über die Grundrechte und -pflichten der Bürger erfüllt. Art. 31 garantiert das Recht der freien Meinungsäußerung in Wort, Schrift und Bild. Erlaubnis beziehungsweise Konzession zur Gründung öffentlicher Druckereien werden dem Staat - als juristischer Person und den Staatsangehörigen von Afghanistan gemäß den Gesetzesvorschriften erteilt (Art.31 Abs. 4). Gründung und Unterhaltung der öffentlichen Anstalten für Rundfunk- und Fernsehsendungen ist dem Staat vorbehalten (Art. 31 Abs. 5.). Das im September 1965 in Kraft getretene neue und liberale Pressegesetz (55 Paragraphen), das zur ungehinderten Entfaltung selbständiger Meinungen und zur Vorbereitung moderner Bildung beiträgt, stellt einen weiteren Fortschritt in Afghanistan dar.

Ein anderer sehr wichtiger Punkt, der im Hinblick auf die Entwicklung in Richtung Modernisierung zu erwähnen bleibt, ist die Gewährung des Rechts zur Gründung politischer Parteien, das zum erstenmal in der Geschichte Afghanistans in der neuen Verfassung verankert ist und somit zur Demokratisierung beiträgt.

In der jüngeren Zeit bis zum Inkrafttreten der neuen Verfassung hatte das afghanische Volk schon demokratische Bestrebungen gezeigt. Auch auf seiten der Regierenden gab es einige, die diesen Bestrebungen Verständnis entgegenbrachten, darunter den prinzlichen Ministerpräsidenten Sardar Schah Mahmud während seiner Amtszeit von 1946 bis 1953. Durch diese zeitweise „Lockerung des Zügels“" bildete sich allmählich im Kreis der Intellektuellen eine Opposition. 1947 wurde in Kandahar eine Vereinigung gegründet, die den Namen „Wisch Zalmjan“ (Erwachende Jugend) trug. Das von der Vereinigung proklamierte Manifest forderte die "Abschaffung schädlicher Gebräuche“, wie unnötig hoher Aufwand bei Hochzeits- und Trauerzeremonien, „den Kampf gegen Korruption und Unterdrückung “ und „die Förderung von Erziehung und Wissenschaft".

Diese Demokratisierungsversuche sahen auch die Gründung politischer Parteien vor; da es jedoch an einer gesetzlichen Grundlage fehlte, blieb der Plan vorläufig ein Wunschtraum. Erst mit dem Inkrafttreten der neuen Verfassung im Jahre 1964 ging er seiner Verwirklichung entgegen.

Nach Art. 32 Abs. 3 haben nun die Staatsbürger Afghanistans das Recht, gemäß den Gesetzesvorschriften politische Parteien zu gründen. Die Zulassung der Parteien wird an folgende Voraussetzungen geknüpft:

1. Ziel und Aktivität der Partei sowie die Grundideen, auf die sich die Parteiorganisation stützt, dürfen nicht im Widerspruch zur Verfassung stehen.

2. Über den inneren Aufbau und die Finanzquellen der Partei soll öffentlich Rechenschaft abgelegt werden.

Eine nach Erfüllung dieser beiden Voraussetzungen zugelassene Partei kann nur aufgelöst werden, wenn sie durch Urteil des Obersten Gerichtshofes für verfassungswidrig erklärt wird (Art. 32 Abs. 4).

Sei es nun auf die einschneidende Bedeutung des Vorhandenseins von Parteien oder auf die geringe Erfahrung der Politiker in den Organisationsfragen von Parteien zurückzuführen - der Entwurf zu einem Gesetz über die Gründung

9 Vgl. Max Klimburg, Afghanistan, das Land im historischen Spannungsfeld Mittelasiens, Wien 1966, S. 73. 
politischer Parteien ist dem Parlament erst Anfang des Jahres 1968 vorgelegt worden. Dieser Entwurf ist inzwischen von beiden Kammern in veränderter Fassung angenommen und seine Koordinierungskommission ist eingesetzt worden ${ }^{\mathbf{1 0}}$.

Wie wichtig das Vorhandensein politischer Parteien in einem Land ist, das sich im Demokratisierungsprozeß befindet, geht aus der Bemerkung eines Marokkaners hervor, die David Apter ${ }^{11}$ wie folgt zitiert: „Marokko kann man keine Demokratie nennen, da ja die ganze Macht nach dem Gesetz in den Händen des Königs konzentriert ist. Trotzdem ist es keine absolute Monarchie, weil andererseits die politischen Parteien im Lande eine markante Rolle spielen. Es ist auch kein Diktaturstaat, weil es dort mehr als eine politische Partei gibt."

\section{Enstehung der neuen Verfassung}

Im 19. und bis zum Anfang des 20. Jahrhunderts mußte Afghanistan viele Krisen im Inneren des Landes im Zusammenhang mit dem Wettstreit der Großmächte um Indien bekämpfen, so daß ihm kaum Zeit blieb, ein geordnetes Rechtswesen zu entwickeln, geschweige denn dem Staat eine geschriebene Verfassung zu geben.

Als 1906 in Iran der Konstitutionalismus proklamiert wurde, verlangte auch das Volk in Afghanistan vom damaligen König Habibullah (1901-1919) die Gewährung einer Staatsverfassung, was jedoch ohne Erfolg blieb ${ }^{12}$.

Am 9. April 1923 bekam Afghanistan seine erste Staatsverfassung von König Amanullah. Diese Verfassung ${ }^{13}$ machte zwar den Staat noch nicht zur konstitutionellen Monarchie - die Staatsform wurde nicht erwähnt - , verbot jedoch erstmalig Sklaverei, Folterung, willkürliche Verhaftung oder grundlose Beschlagnahme von Eigentum $^{14}$. Wegen einiger für die damalige Zeit verfrühter Neuerungen, wie zum Beispiel der Trennung von Religion und Staat und der völligen Emanzipation der Frauen, wurde jedoch der König von der Geistlichkeit (den Ulama) des Landes beim Volk in Mißkredit gebracht ${ }^{15}$. Das Fazit war die Abdankung des Königs und ein darauffolgender neunmonatiger Bürgerkrieg im Jahre 1929.

Am 31. Oktober 1931 bekam Afghanistan seine zweite Verfassung. Diese Verfassung bestand aus 110 Artikeln ${ }^{16}$, zu deren Ausarbeitung eine verfassungsgebende Versammlung, genannt „Loja Djerga“"17, einberufen worden war. Sie konnte zwar das Land aus den Folgen des schrecklichen Bürgerkrieges retten, die Staatsform blieb jedoch streng autokratisch. Im gesamten Text dieser Verfassung stand eine allumfassende Kompetenz des Königs im Vordergrund" ${ }^{18}$. So kam es, daß das Land drei Jahrzehnte nacheinander von drei prinzlichen Ministerpräsidenten ${ }^{19}$ und fast

$10 \mathrm{Vgl}$. Elisabeth Malle, Wandlungen in Afghanistan, in: „Indo-Asia“ (Stuttgart) 1968, S. 169.

11 A. a. O., S. 180 (englisch).

12 Vgl. Klimburg, a. a. O., S. 57.

13 Wegen des Textes dieser Verfassung vgl. Josef Schwager, Die Entwicklung Afghanistans als Staat und seine zwischenstaatlichen Beziehungen, Dissertation, Leipzig 1932. Italienische Úbersetzung des Textes in D. M. 1931, S. 276.

14 Vgl. Klimburg, a. a. O., S. $62 / 63$

15 Vgl. W. K. Fraser-Tytler, Afghanistan, A Study of Political Development in Central and Southern Asia, Third Edition, London, Oxford University Press, New York-Toronto 1967, S. $212 / 213$.

16 Der. Text dieser of $t$ abgedruckten Verfassung befindet sich z. B. in englischer Sprache bei Helen MillerDavis, Constitutions, electoral laws, treaties of States in the Near and Middle East, Durham, N. C., 1953, S. $4 \mathrm{ff}$

17 Seit Mitte des 18. Jahrhunderts berief der König des Landes einen aus den Altesten der Stämme bestehenden Rat, genannt "Djerga“, zu sich, um mit ihm die wichtigsten Probleme der Bevölkerung zu besprechen. Betraf jedoch ein "Problem alle Provinzen gemeinsam und somit den Staat, so wurde vom König die sogenannte „Loja Djerga“, das heißt „Die Große Versammlung“, einberufen.

18 Vgl. Ata Mohammed Nursai, Materialien und Wege der staatlichen Verwaltungsstruktur in Afghanistan, jur. Dissertation, Köln 1963, S. 28.

19 Vgl. Klaus Ackermann, Stille Revolution in Afghanistan, in: Außenpolitik (Stuttgart) 1965, S. 33. 
ein Jahrzehnt von einem prinzlichen Außenminister ${ }^{20}$ regiert wurde. Alle in der Verfassung von 1931 verankerten Freiheiten wurden in der Praxis wieder stark eingeschränkt. Politische Parteien blieben verboten. Jede Kritik an der Regierung bedeutete Kritik an der königlichen Familie und damit einen Verstoß gegen das Ansehen des Staates. Man konnte in der ausländischen Presse Schlagzeilen lesen wie „In Kabul herrscht ein Familienkonzern“21. Im Zuge der Entwicklung und ständigen Kontaktnahme mit der Außenwelt, sei es durch die Ausbildung der Führungskräfte des Landes aus der Bürgerschicht im Ausland oder durch den modernen Unterricht der eigenen Lehrkräfte und derer aus befreundeten Staaten an afghanischen Hochschulen und schließlich durch die Handelsbeziehungen mit den Nachbarstaaten und den Staaten des Nahen Ostens wurde allmählich in Afghanistan der Boden für eine Reform tiefgreifender Art reif. So sah sich der gegenwärtige König des Landes, Mohammed Zahir Schah, ein Mann, der in Frankreich studiert hat ${ }^{22}$ und ein gutes politisches Feingefühl besitzt, schließlich einer ziemlich prekären Situation gegenüber. Es mußte etwas geschehen und es geschah etwas. Nach einer langen Sitzung der königlichen Familie hinter geschlossenen Türen trat im März 1963 der letzte prinzliche Ministerpräsident Sardar Mohammed Da'ud zurück. Der König beauftragte den aus der Bürgerschicht stammenden Physiker Dr. Mohammed Jussof mit der Bildung eines neuen Kabinetts. Nunmehr standen der König und der neue Ministerpräsident an der Spitze der Bestrebungen des Landes nach Fortschritt und Modernisierung.

Eine Frage, die sich an dieser Stelle erhebt, ist, ob und inwiefern bisher in Afghanistan unter den erwähnten Voraussetzungen eine personale Ordnung etwa in feudalistischer Form geherrscht hat. Ähnlich dem, was viele europäische Staaten vom Mittelalter über die verschiedenen Stadien des Absolutismus bis hin zur Aufklärung, den Zeiten der konstitutionellen Monarchie und der Republik erlebt haben, sind auch Afghanistan und andere vorder- und mittelasiatische Staaten ihren Weg von einer feudalistischen zu moderneren Herrschaftsformen gegangen. Die personale Herrschaftszeit in Afghanistan reicht etwa bis zum Anfang des 20. Jahrunderts. Der erste Versuch des Volkes zur Konstitutionaliserung des Staates im Jahre 1908 scheiterte - wie schon erwähnt - an der ablehnenden Haltung des damaligen Königs Habibullah. 1923 erhielt das Volk die erste Verfassung. Sie wurde 1931 von einer zweiten abgelöst. Die Staatsform blieb jedoch bis 1964 eine wenig eingeschränkte Monarchie, in der der König noch die wichtigsten Rechte selbst ausübte. Erst nach dem Inkrafttreten der neuen Verfassung von 1964 kann sich Afghanistan der Staatsform einer wirklich konstitutionellen Monarchie erfreuen ${ }^{23}$.

Die Vorgänge im Zusammenhang mit der Verfassungsänderung könnten andererseits die Schlußfolgerung nahelegen, die neue Verfassung versuche den Staat zur Institution zu machen. Obwohl nach näherer Betrachtung des Verfassungstextes ${ }^{24}$ diese Schlußfolgerung sich nicht ohne weiteres ziehen läßt, ist zumeist eine Grundlage dafür in der Verfassung geschaffen worden, indem jedem Bürger Gelegenheit gegeben wird, an der Gestaltung des politischen Lebens im Lande mitzuwirken

20 Vgl. Karl Otto Hondrich, Verfassungsentwicklung, politische Stabilität, sozialer Wandel - Die Modernisierung des traditionellen politischen Systems in Afghanistan, in: Verfassung und Verfassungswirklichkeit, Band 1, Jahrbuch 1966, Köln und Opladen, S. 222, 223.

21 Thilo Bode, in "Die Welt", Nr. 279, vom 29. Oktober 1960, S. 3.

22 Vgl. Afghanistan at a Glance, Veröffentlichung der Kabul Times Publishing Agency, Kabul, März 1967, Vorwort.

23 Als eines der markantesten Merkmale dieser neuen Staatsform ist die Berufung von Fachleuten in hohe Regierungsämter anzusehen. Vgl. auch Hondrich, a. a. O., S. 241.

24 Z. B. hat der König das Recht, den Ministerpräsidenten zu ernennen (Art. 9 Abs. 11) und die Entscheidung über den Krieg und Frieden zu treffen (Art. 9 Abs. 2). 
(Art. 41 ff.). Wieweit jedoch diese Idee in der Zukunft Wirklichkeit werden wird, hängt davon ab, wie schnell sich ein politisches Bewußtsein in der breiteren Schicht der Bevölkerung heranbilden läßt. Was dem hemmend engegenwirken könnte, ist, wie auch Hondrich ${ }^{25}$ zutreffend bemerkt, eine tief in der Tradition wurzelnde Gesellschaft, in der die Menschen zur Hinnahme eines als unabänderlich empfundenen Schicksals erzogen werden, und in der jeder gemäß seiner Herkunft Status und Rolle „zugewiesen“ bekommt. Als weiteres retardierendes Moment ist die Tatsache zu werten, daß obwohl durch die neue Verfassung alle Angehörigen der königlichen Familie aus den Staatsämtern entfernt worden sind, die Machtstellung des König doch weiterhin bestehen bleibt, indem laut Art. 6 „der König die nationale Herrschaft in Afghanistan darstellt".

Etwa Mitte des Jahres 1963 hatte der neue Premierminister Dr. Jussof die Ausarbeitung einer neuen Verfassung angekündigt. Es sollte dazu eine Verfassungskommission im Justizministerium gebildet werden. Nach Fertigstellung des Entwurfes zur neuen Verfassung sollte der Große Ältestenrat - Loja Djerga - als Nationalversammlung einberufen werden und über den Entwurf abstimmen.

Die Verfassungskommission begann sogleich unter dem Vorsitz des Justizministers Madjruh ihre Arbeit und sammelte eine Reihe historischer und wissenschaftlicher Werke über die Entwicklung der Regierungsform in Afghanistan und über die Vorgänge in den Verhandlungen verschiedener "Großer Versammlungen“, die von der Gründung des Staates unter jetziger Bezeichnung im Jahre 1747 bis zur Gegenwart einberufen worden waren. Ferner wurde der Verfassungskommission durch die afghanischen diplomatischen Vertretungen im Ausland eine Reihe von Texten zu den Verfassungen befreundeter Staaten zur Verfügung gestellt. Zum Zwecke der Beratung durch einen europäischen Rechtsexperten schickte Frankreich auf die Bitte der afghanischen Regierung ein Parlamentsmitglied, das bereits mit dem Rechtssystem der islamischen Länder Nordafrikas vertraut war, nach Kabul. Die führenden Persönlichkeiten des Landes auf den Gebieten der Politik, der Religions-, Wirtschafts- und Rechtswissenschaft wurden um ihre Meinungen und um Ratschläge gebeten.

Nach eingehendem Studium aller gesammelten Unterlagen und Prüfung der Meinungsäußerungen und Ratschläge stellte dann die Verfassungskommission einen neuen Verfassungstext zusammen. Der einberufene Große Altestenrat - Loja Djerga -, der vom 9. bis 19. September 1964 in Kabul tagte, nahm die neue Verfassung einstimmig an ${ }^{26}$, die am 1. Oktober 1964 vom König unterzeichnet wurde. Nunmehr konnte man in der ausländischen Presse Schlagzeilen lesen wie „Kabul's New Men“27 oder „Eine Demokratie entsteht in Zentralasien“28.

Als Vorsitzender der Verfassungskommission hob der Justizminister die Berücksichtigung folgender Leitgedanken bei der Ausarbeitung der neuen Verfassung hervor:

a) Wahrung des Gleichgewichts zwischen den Interessen der Gesellschaft und den Rechten und Interessen des einzelnen,

b) Bildung eines selbständigen und wirksamen Rechtsprechungsapparates sowie Stärkung der Legislative,

c) Befestigung einer wirksamen und verantwortungsbewußten Verwaltung,

\footnotetext{
25 A. a. O., S. 211, 213, 241.

26 Über die Entstehung der neuen Verfassung vgl. „Nassim-e-Bakhter“ (das Monatsnachrichtenblatt des Afghanisch-Europäischen Kulturamtes in München), Jahrgang XI, Nr. 12, vom 20. März 1964, S. 15-18 (in afghanischer Dari-Sprache).

27 The Economist, London, vom 6. Februar 1965, S. 523/524.

28 Elisabeth Malle, in: „Ưbersee-Rundschau“, Hamburg, Juni 1967, S. 23-25.
} 
d) Grundsätzliche Beibehaltung der Prinzipien des Islam,

e) Rolle des Monarchen als Mittelpunkt des Gleichgewichts zwischen den drei Staatsgewalten,

f) Regelung der Frage der Thronfolge.

\section{Inhaltsübersicht der neuen Verfassung mit besonderer Berücksichtigung der Kapitel über die Gesetzgebung, Verwaltung und Rechtsprechung}

Die neue Verfassung besteht aus einer Präambel und 128 Artikeln, die in elf Kapitel gegliedert sind.

In der Präambel wird unter anderem zum Ausdruck gebracht: „Zum Zwecke der Ordnung des nationalen Lebens in Afghanistan, ..., zum Zwecke der Sicherung von Gerechtigkeit und Gleichheit, zur Verwirklichung politischer, wirtschaftlicher und sozialer Demokratie, ... und zum Zwecke der gleichmäßigen (!) Entwicklung des Landes in allen Lebensbereichen erlassen wir, Afghanistans Bürger, unter nationaler Führung seiner Majestät des Königs, diese Verfassung für uns und für unsere zukünftigen Generationen."

Das erste Kapitel enthält Bestimmungen über den Staat (Art. 1-5). Das zweite ist dem König gewidmet (Art. 6-24). Die Grundrechte und -pflichten sind im dritten Kapitel verankert (Art. 25-40). Das vierte gibt Aufschluß über die Zusammensetzung des Parlaments (Art. 41-77). Im fünften werden die Vorschriften über die Einberufung der Nationalversammlung - Loja Djerga - niedergelegt (Art. 78-84). Die Regierung wird in Kapitel 6 behandelt (Art. 85-96). Das siebte befaßt sich mit der Rechtsprechung (Art. 97-107). Mit den Fragen der Verwaltung beschäftigt sich das achte (Art. 108-112). Der Ausnahmezustand wird im neuten erörtert (Art. 113-119). Über die Möglichkeiten zur Änderung der Verfassung ist in Kapitel $10 \mathrm{zu}$ lesen (Art. 120-122). Schließlich gibt Kapitel 11 Übergangs- und Schlußbestimmungen (Art. 123-128).

Die wichtigsten Punkte der Verfassung, die sie insbesondere von der Verfassung von 1931 unterscheiden, sind: Umwandlung der Staatsform von einer absoluten in eine konstitutionelle Monarchie, Gewaltentrennung, Verbot für Angehörige der königlichen Familie, außer Mitglied in einer politischen Partei zu werden, das Amt des Premierministers oder eines Minsters sowie das Amt eines Mitgliedes des Obersten Gerichtshofes und des Parlaments zu bekleiden.

Weiterhin werden außer den Grundsätzen der „Gleichheit aller Staatsbürger vor dem Gesetz“ und „nulla poena sine lege“, die Wahrung der Würde des Menschen, der Schutz des Lebens und der Gesundheit, die Unverletzlichkeit der Wohnung, des Brief- und Postgeheimnisses und die Freizügigkeit, Glaubens-, Versammlungsund Koalitionsfreiheit, das Recht der freien Meinungsäußerung, der Berufswahl und der Arbeit garantiert; schließlich wird das Verbot der Zwangsarbeit ausgesprochen.

Die Entrichtung von Steuern und Abgaben, die Ableistung eines Militärdienstes, die Befolgung der Vorschriften der Verfassung und der übrigen Gesetze, Treue und Ehrfurcht gegenüber dem König, Wahrung der Belange des Staates und Beteiligung an der Gestaltung des öffentlichen Lebens bilden dagegen die Pflichten jedes Staatsbürgers. 
Die Gesetzgebung

Die Gesetzgebungskompetenz in Afghanistan steht dem Parlament als dem Repräsentanten des ganzen Volkes zu. Afghanistans Bürger nehmen durch das Parlament am politischen Leben des Landes teil (Art. 41).

Das Parlament besteht aus zwei Kammern:

a) dem Nationalrat, genannt „Wolossi Djerga“, dessen Mitglieder für die Dauer von vier Jahren, die auch eine Legislaturperiode ist, durch freie, allgemeine, geheime und unmittelbare Wahlen vom Bürger gewählt werden (Art. 44).

b) dem Staatsrat oder Senat, genannt „Meschrano Djerga“, dessen Mitglieder zu einem Drittel vom König für die Dauer von fünf Jahren ernannt werden. Ein weiteres Drittel wird aus der Mitte der Regionalversammlungen der Provinzen für die Dauer von drei Jahren und das letzte Drittel durch das Volk nach Provinzen auf dem Wege von freien, geheimen, allgemeinen und unmittelbaren Wahlen für die Dauer von vier Jahren gewählt (Art. 45).

Der Nationalrat wählt aus der Mitte seiner Mitglieder eine Person als Präsidenten. Der Präsident des Senats wird aus der Mitte seiner Mitglieder vom König ernannt (Art. 60).

Ein Gesetz tritt nach der gemeinsamen Billigung durch die beiden Kammern und der Unterzeichnung durch den König in Kraft (Art. 69) ${ }^{29}$.

Die Verwaltung

Afghanistans Verwaltung ist auf dem Grundsatz des Zentralismus aufgebaut. Die Zentralverwaltung ist in verschiedene Ressorts unterteilt, an deren Spitze jeweils ein Minister steht. Die Einheit der Regionalverwaltung ist die Provinz (Art. 108), mit einem von der Zentralregierung ernannten Gouverneur an der Spitze. In jeder Provinz wird außerdem ein Provinzrat gebildet. Dieser Provinzrat ist gewissermaßen ein Kontrollorgan gegenüber dem Gouverneur und geht aus freien, allgemeinen, geheimen und unmittelbaren Wahlen hervor. Er wirkt bei den Entwicklungsplänen der Zentralregierung, sowie bei denen der Provinzregierung mit (Art. 109). Zur Regelung der städtischen Angelegenheiten werden Stadtverwaltungen gebildet. Die Stadtverwaltung besteht aus drei Organen, nämlich:

Der „Madjlis“ genannten Stadtverordnetenversammlung, die jährlich einmal zusammentritt, dem Ausschuß („Andjuman“), der die laufenden Geschäfte abwickelt und die entsprechenden Entscheidungen trifft, und dem Bürgermeister.

Die Satzungen der Stadtverwaltung werden auf dem Wege der freien, allgemeinen und geheimen Abstimmung beschlossen (Art. 111). Der von der Zentralregierung eingesetzte Bürgermeister und die Mitglieder der weiteren Gemeindeorgane sind an die Satzungen gebunden. Dieser Status der "Gemeinde“ ist jedoch besonderen, verwaltungsmäßig hervorgehobene Städten vorbehalten ${ }^{30}$.

\section{Die Rechtsprechung}

Die neue Verfassung sieht in ihrem Kapitel 7 die Unabhängigkeit der Rechtsprechung vor und proklamiert die Zusammenfassung aller Gerichte im Rahmen einer neugeformten Organisation, die den Namen "Qassa'“ (Judikative) trägt ${ }^{31}$. Um die bedeutsamen Neuerungen im Bereich der Rechtsprechung richtig würdigen $\mathrm{zu}$ können, ist es unumgänglich, die Geschichte der afghanischen Gerichtsbarkeit

29 Vgl. wegen der Gesetzgebung ferner Hondrich, a. a. O., S. 230-233.

30 Vgl. Nursai, a. a. O., S. 51/52.

31 Vgl. "Qassa', die Veröffentlichung des Justizministeriums anläßlich der Eröffnung des Obersten Gerichtshofes" am 12. Oktober 1967, Kabul 1967, S. $10 \mathrm{ff}$. (in Dari-Sprache). 
mit einigen Sätzen zu skizzieren. Über das Rechtswesen im vorislamischen Afghanistan berichtet der chinesische Mönch und Historiker Hsüan Chuang (um 630 n. Chr.) vom Osten des Landes und von der Umgebung von Kabul, daß diejenigen, die die Sicherheit des Staates und das Vermögen der Bürger gefährdet hatten, $z u$ entsprechenden Strafen verurteilt wurden. Im Süden und Westen des Landes sollen die Regeln der zoroastrischen Lehre zur Vergeltung von Straftaten angewandt worden sein.

Um die Zeit, als der Islam nach Afghanistan vordrang, und danach genoß das Amt des Richters dort großes Ansehen. In der berühmten Moschee „'Arus-alFalak" (Braut des Weltalls) in Ghazni ließ zum Beispiel Sultan Mahmud von Ghazna (997-1030 n. Chr.) zum Zwecke der „Qassa“" (Rechtsprechung) besondere Nischen mit Hilfspersonal für Richter einrichten.

Ein oberstes Gericht wurde während der Regierungszeit von Emir Abdul Rahman (1880-1901) in Kabul auf Grund der Beschwerden der Rechtsuchenden über die schikanöse Verhandlungsweise der Gerichte gegründet.

Das Dreistufensystem, das heißt das Verfahren im ersten Rechtszuge, in der Berufung und in der Revision, wurde während der Regierungszeit von König Amanullah (1919-1929) eingeführt. Während der Regierungszeit von König Mohammed Nader (1929-1933) bekam die Judikative zum erstenmal eine gesetzliche Form, indem der Verfassung von 1931 ein besonderes Kapitel unter dem Titel "Gerichte“ eingefügt wurde.

Weitere Vervollkommnungen erfuhr das Rechtswesen während der Regierungszeit des gegenwärtigen Königs Mohammed Zahir Schah ab 1933.

Nach Art. 97 der neuen Verfassung ist die "Judikative“ ein selbständiges Organ des Staates und nimmt ihre Aufgaben neben den Organen der Gesetzgebung und der Verwaltung wahr. Die "Judikative" besteht aus einem Obersten Gerichtshof, genannt "Sterra Mahkama“, und anderen Gerichten, deren Anzahl durch Gesetz bestimmt wird. Die Zuständigkeit des Obersten Gerichtshofes umfaßt alle Arten von Rechtsstreitigkeiten, bei denen natürliche oder juristische Personen einschließlich des Staates als Kläger oder Angeklagte auftreten und gemäß den Gesetzesvorschriften den Obersten Gerichtshof anrufen (Art. 98). Dessen Richter werden auf Vorschlag des obersten Richters vom König ernannt (Art. 99). Der Oberste Gerichtshof besteht aus neun Mitgliedern, die vom König ernannt werden (Art. 105). Die Mitglieder des Obersten Gerichtshofes müssen das fünfunddreißigste Lebensjahr vollendet haben, ferner die Fähigkeit besitzen, gemäß Art. $46 \mathrm{der}$ Verfassung ${ }^{32}$ als Parlamentsmitglied gewählt $\mathrm{zu}$ werden und über genügende Kenntnis der Rechtsvorschriften, der nationalen Ziele und der Rechtsordnung des Landes verfügen. Der König ernennt aus der Mitte der Mitglieder des Obersten Gerichtshofes den obersten Richter, dessen Alter zwischen 40 und 60 Jahren liegen soll (Art. 105 Abs. 2). Art. 106 enthält Vorschriften über das Gerichtsverfahren gegen die Mitglieder des Obersten Gerichtshofes, die sich einer Straftat während ihrer Amtstätigkeit schuldig gemacht haben. Der Oberste Gerichtshof ist Afghanistans höchste richterliche Instanz (Art. 107). Die Aufgaben des Obersten Gerichtshofes sind außer der Rechtsprechung der Erlaß eines Gerichtsverfassungsgesetzes, die Erstellung des Haushaltsplanes für die "Judikative" und die Kontrolle über seine Einhaltung.

32 Die Mitglieder des Parlaments müssen nach Art. 46 folgende Voraussetzungen erfüllen:

1. mindestens seit zehn Jahren afghanische Staatsangehörige sein,

2. nach Inkrafttreten der Verfassung (von 1964) nicht die bürgerlichen Ehrenrechte durch Urteil des Gerichtes verloren haben, 


\section{Bedeutung der Judikative für die zukünftige Entwicklung des Landes}

Wie aus den bisherigen Ausführungen ersichtlich, erhält die Judikative in Zukunft eine besondere Bedeutung, und zwar aus den folgenden Gründen:

Die Praxis der Gerichte beruhte bisher zum größten Teil auf dem islamischen Recht und wurde vom "Qa'zi“, dem Absolventen einer Theologieschule, ausgeübt. Die Prozesse wurden ohne öffentlichen Ankläger geführt. Man war der Meinung, daß durch eine Straftat erstens das Recht Gottes („Haq-ullah“) und zweitens das Rechtsgut des Bürgers („Haq-ul'abd“) verletzt worden ist. Der Privatkläger versuchte den Angeklagten durch Aussagen von zwei Zeugen für schuldig zu erklären. Der Angeklagte hatte seinerseits die Möglichkeit, durch Aussagen von zwei Zeugen die Glaubwürdigkeit der Zeugenaussagen des Gegners in Frage zu stellen. Auf Grund der Zeugenaussagen, eines eventuellen Geständnisses und nach den geltenden Gesetzen fällte dann der Richter das Urteil ${ }^{33}$. Nach dem Inkrafttreten der neuen Verfassung ist jedoch dieser alte Brauch weitgehend ersetzt worden durch ein Verfahren, wie es in jeder fortschrittlichen Gesellschaft geführt wird, nämlich durch die Erhebung der öffentlichen Anklage. Man ist, wie in der westlichen Rechtsordnung, zu der Theorie übergegangen, daß durch eine Straftat erstens das Recht des Bürgers und zweitens das der Öffentlichkeit betroffen wird. Die Anklage wird nunmehr von dem öffentlichen Ankläger, also dem Staatsanwalt, erhoben. Der Angeklagte hat das Recht, für seine Verteidigung einen Rechtsanwalt zu nehmen. Der Richter fällt auf Grund eines Plädoyers des Staatsanwalts und des Verteidigers und eventuell eingebrachter Beweise das Urteil. Die Richter sind genauso wie früher vom Staat fest angestellt und werden von ihm besoldet.

Nach Abschnitt 1 Kapitel 1 des Gesetzes über die Organisation und Zuständigkeit der Judikative (Gerichtsverfassungsgesetz) vom November 1964 werden die Gerichte zusammengefaßt in

a) Allgemeine Gerichte; dies sind:

Der Oberste Gerichtshof, die Berufungsgerichte, die Provinzgerichte und die Gerichte im ersten Rechtszug

b) Besondere Gerichte; dies sind:

Handelsgerichte des ersten Rechtszuges in Kabul, das Gericht des ersten Rechtszuges für die öffentliche Sicherheit in Kabul, die Jugend- und schließlich die Arbeitsgerichte.

Im Abschnitt 1 Kapitel 2 erklärt das erwähnte Gesetz die Gerichte für alle Straftaten $(\$ 24)$ beziehungsweise alle Streitigkeiten aus dem Zivil- und Handelsgesetz ( $(25)$ für zuständig. Diese Zuständigkeit erstreckt sich auf die folgenden Rechtsbeziehungen:

a) aus dem Zivil- und Handelsrecht, die zwischen Staat und natürlichen oder juristischen Personen über bewegliche und unbewegliche Sachen entstehen,

b) aus erlittenen Schäden, die durch die Tätigkeit der Regierung oder der Verwaltung entstehen,

c) aus den Schäden, die durch Verträge entstehen, die mit den staatlichen Behörden und Institutionen abgeschlossen werden,

d) zwischen natürlichen und juristischen Personen,

3. des Lesens und Schreibens kundig sein und

4. als Mitglieder des Nationalrates das 25. und als Mitglieder des Senats das 30 . Lebensjahr vollendet haben.

33 Vgl. Klimburg, a. a. O., S. 103; Hondrich, a. a. O., S. 240. 
e) über anderen Angelegenheiten, wenn die Beschlußfassung über sie nach der Verfassung und den Staatsgesetzen einem Gericht überlassen wird oder überlassen worden ist.

Abschnitt 2 des Gerichtsverfassungsgesetzes befaßt sich mit den Anstellungsfragen der Richter ( $\mathbb{S}$ 38-42). In Strafsachen und auf einigen Gebieten des Zivilrechts zum Beispiel Erb- und Familienrechts, wurde bisher immer noch das Richteramt von den Absolventen der theologischen Hochschule wahrgenommen. In anderen, säkularisierten Rechtsgebieten wie zum Beispiel Handels-, Arbeits- und Jugendgerichtsbarkeit werden neuerdings Richter mit dem Abschlußexamen der juristischen Fakultät der Universität Kabul angestellt. Es ist auch das System eines Gerichtspraktikums nach dem Hochschulexamen und vor der endgültigen Anstellung eingeführt worden.

Rechts- und Staatsanwälte können ebenfalls nach Erfüllung der erforderlichen Voraussetzungen, die Mitglieder des Parlaments und des Gemeindeausschusses jedoch nach Ablauf von fünf Jahren in dem Ort, den sie beim Parlament oder bei der Gemeinde vertreten haben, mit dem Richteramt betraut werden.

Wie aus der Umorganisation des Gerichtswesens ersichtlich, werden durch das ständige Anwachsen von Handel, Verkehr, Wirtschaft und Industrie die genannten Gerichte in steigendem Maße in Anspruch genommen, was wiederum den Erlaß neuer Gesetze und die Schaffung neuer Arbeitsplätze für neues Personal erfordert.

\section{Schlußbetrachtung}

„Afghanistan steht an der Schwelle einer neuen demokratischen Epoche. Nach vielen Jahren des inneren Friedens ist das Land im Stadium des tiefgreifenden Aufbruchs in die ,Neuzeit'. Afghanistan wird daher in zunehmendem Maß von inneren Krisen und Spannungen heimgesucht werden, wie dies andere Staaten bereits durchgemacht haben oder soeben durchmachen." Diese düsteren Worte stehen im Vorwort einer Publikation über Afghanistan, die auf Grund des vierjährigen Aufenhalts ihres Verfassers, Herrn Max Klimburg, im Lande im Auftrage der österreichischen UNESCO-Kommission entstanden ist.

Wie wir gesehen haben, am Beginn dieser Epoche haben sowohl der König als auch die Bevölkerung des Landes tatkräftig mitgewirkt.

Die Bestrebungen nach Fortschritt und Modernisierung finden ihren Niederschlag einmal in der Präambel zu der neuen Verfassung und dann in der neuen Verfassung selbst, in der außer Einführung vieler neuer Prinzipien, wie zum Beispiel der Gewaltentrennung - unter besonderer Hervorhebung der Judikative - , die Grundfreiheiten des einzelnen Bürgers garantiert werden. Es sind neue und umfangreiche Möglichkeiten des gesellschaftlichen Aufstieges für jedermann, auch für die Frauen, im sozialberuflichen, wirtschaftlichen und politischen Bereich geschaffen worden. Den Fortschrittsbemühungen entgegenstehende Hemmnisse allmählich beseitigen zu helfen, soll die Aufgabe der neuen Verfassung sein. Sie ist nämlich als ein guter Leitfaden für politisch Handelnde zu betrachten, die in immer größerer Zahl in das Leben des Staates eintreten müßten.

$\mathrm{Da}$ dies geschehen wird, ist indes zu erwarten, denn das Wort Montesquieus dürfte sich auch hier bewahrheiten, daß man nämlich in einem Land, in dem die Verfassung Anteil an der Staatsführung und am politischen Leben gewährt; viel von Politik sprechen wird. 